\title{
Beyond Maxi-Consistent Argumentation Operators ${ }^{\star}$
}

\author{
Srdjan Vesic Leendert van der Torre \\ Computer Science and Communication \\ University of Luxembourg \\ srdjan.vesic@uni.lu, leon.vandertorre@uni.lu
}

\begin{abstract}
The question whether Dung's abstract argumentation theory can be instantiated with classical propositional logic has drawn a considerable amount of attention among scientists in recent years. It was shown by Cayrol in 1995 that if direct undercut is used, then stable extensions of an argumentation system correspond exactly to maximal (for set inclusion) consistent subsets of the knowledge base from which the argumentation system was constructed. Until now, no other correspondences were found between the extensions of an argumentation framework and its knowledge base (except if preferences are also given at the input of the system). This paper's contribution is twofold. First, we identify four intuitive conditions describing a class of attack relations which return extensions corresponding exactly to the maximal (for set inclusion) consistent subsets of the knowledge base. Second, we show that if we relax those conditions, it is possible to instantiate Dung's abstract argumentation theory with classical propositional logic and obtain a meaningful result which does not correspond to the maximal consistent subsets of the knowledge base used for constructing arguments. Indeed, we define a whole class of instantiations that return different results. Furthermore, we show that these instantiations are sound in the sense that they satisfy the postulates from argumentation literature (e.g. consistency, closure). In order to illustrate our results, we present one particular instantiation from this class, which is based on cardinalities of minimal inconsistent sets a formula belongs to.
\end{abstract}

\section{Introduction}

The question how to reason in presence of inconsistency is on of the keywords of logic and artificial intelligence. A notable example are paraconsistent logics [11] where one is able to draw some (but not all) conclusions from an inconsistent set of formulae. As another example take belief revision, belief merging or voting [8]. Generally speaking, an inference relation is a way to go from a (possibly inconsistent) knowledge base to a set of subsets of that knowledge base. For example, given a knowledge base $\{\varphi, \neg \varphi \wedge$ $\psi\}$, an inference relation could return two sets: $\{\varphi\}$ and $\{\neg \varphi \wedge \psi\}$. One of the simplest inference relations is a function returning the set of all maximal (for set inclusion) consistent subsets of a knowledge base. It has been shown [6] that the result obtained

\footnotetext{
* SV was funded by the National Research Fund, Luxembourg. His work was carried out during the tenure of an ERCIM "Alain Bensoussan" Fellowship Programme. This Programme is supported by the Marie Curie Co-funding of Regional, National and International Programmes (COFUND) of the European Commission.
} 
by this inference relation can be also obtained by an instantiation of Dung's abstract argumentation theory [7]. Namely, when direct undercut is used as attack relation on the set of all the arguments built from a knowledge base, then stable extensions of the resulting argumentation framework correspond exactly to the set of maximal consistent subsets of the knowledge base. This is the first result which shows that Dung's abstract argumentation theory can be instantiated in a way to capture an inference relation.

An important question is whether Dung's theory can be used as a general framework for nonmonotonic logic, and if so, which class of inference relations can be studied as instances of Dung's theory. Indeed, only a very small fragment of logics has been represented in such a way. This may also not be very surprising, given the richness of the logic literature and the strong constraints imposed by Dung's theory. This raises two important questions for the community. First, which class of logics can be captured by Dung's theory? Second, how to generalize Dung's theory? In this paper we address the first question, whereas the second question is a part of our long term research agenda.

The starting point of our work is to note that since the first result [6] showing how to capture an inference relation in Dung's theory, not much work has been done in this direction. Indeed, no "reasonable" logic-based instantiations of Dung's abstract theory were found that capture another inference relation. By "reasonable", we mean that they satisfy at least some basic postulates proposed for instantiated argumentation frameworks [5] like consistency, closure, and so on.

The challenges of this paper are: First, is it possible to define conditions that characterize the circumstances when a semantics returns maximal consistent subsets under subset relation? Second, how to define a class of attack relations in terms of the knowledge base such that the stable extensions of the obtained argumentation framework do not correspond to exactly to the maximal for set inclusion consistent subsets of the knowledge base? Third, how to ensure that those instantiations of Dung's theory still return a reasonable result?

The layout of this paper is as follows: After introducing the notions of argumentation framework and formally defining its logic-based instantiations (Section 2), we identify four conditions describing a class of attack relations returning extensions corresponding to maximal consistent subsets of a knowledge base (Section 3). Then, we show that if two conditions are dropped, it is possible to instantiate Dung's abstract argumentation theory in a meaningful way and obtain a substantially different result (Section 4). The last section concludes and reviews questions left for future work. The proofs are omitted due to the space restrictions.

\section{Dung's Abstract Argumentation Theory and its Instantiation with Classical Propositional Logic}

In this section, we present the most common way of instantiating Dung's abstract argumentation theory [7] with classical propositional logic. $\mathcal{L}$ denotes the set of wellformed formulae, $\vdash$ stands for classical entailment, and $\equiv$ for logical equivalence. We denote by $\Sigma$ a finite set of classical propositional formulae from which arguments are constructed. We use the notation $\operatorname{MC}(\Sigma)$ for the set of all maximal (for set inclusion) 
consistent subsets of $\Sigma$, and $\operatorname{Min} \operatorname{Conf}(\Sigma)$ for the set of minimal (for set inclusion) inconsistent subsets of $\Sigma$. A formula $\varphi$ is called a free formula of a knowledge base $\Sigma$ if and only if $\varphi$ does not belong to any minimal (for set inclusion) inconsistent subset of $\Sigma$. A logical argument is defined as a pair (support, conclusion).

Definition 1 (Argument). Let $\mathcal{L}$ be a classical propositional language with $\vdash$ its associated logical consequence, let $\Sigma \subseteq \mathcal{L}$ and $\alpha \in \mathcal{L}$. An argument is a pair $(\Phi, \alpha)$ such that $\Phi \subseteq \Sigma$ is a minimal (for set inclusion) consistent set of formulae such that $\Phi \vdash \alpha$.

Example 1. Let $\Sigma=\{\varphi, \varphi \rightarrow \psi, \omega\} .(\{\varphi, \varphi \rightarrow \psi\}, \psi),(\{\varphi \rightarrow \psi\}, \neg \varphi \vee \psi)$ and $(\{\varphi, \psi\}, \varphi \leftrightarrow \psi)$ are some of the arguments that can be constructed from $\Sigma$.

For an argument $a=(\Phi, \alpha)$, we write $\operatorname{Supp}(a)=\Phi$ to denote its support and $\operatorname{Conc}(a)=\alpha$ to denote its conclusion. For a set of arguments $\mathcal{E}$, we denote by $\operatorname{Concs}(\mathcal{E})$ the set of conclusions of all the arguments from $\mathcal{E}$. In other words, $\operatorname{Concs}(\mathcal{E})=\{\operatorname{Conc}(a)$ $\mid a \in \mathcal{E}\}$. For a given set of formulae $S \subseteq \mathcal{L}$, we denote by $\operatorname{Arg}(S)$ the set of arguments constructed from $S$. Formally, $\operatorname{Arg}(S)=\{a \mid a$ is an argument and $\operatorname{Supp}(a) \subseteq S\}$. Let $\operatorname{Arg}(\mathcal{L})$ denote the set of all arguments that could be made from propositional logic formulae. For a given set of arguments $\mathcal{E}$, we denote $\operatorname{Base}(\mathcal{E})=\bigcup_{a \in \mathcal{E}} \operatorname{Supp}(a)$. Now we provide a definition of argumentation framework.

Definition 2 (Argumentation framework). An argumentation framework is a pair $(\mathcal{A}, \mathcal{R})$ where $\mathcal{A} \subseteq \operatorname{Arg}(\mathcal{L})$ is a set of arguments and $\mathcal{R} \subseteq \mathcal{A} \times \mathcal{A}$ a binary relation. For each pair $(a, b) \in \mathcal{R}$, we say that a attacks $b$. We also sometimes use notation $a \mathcal{R} b$ instead of $(a, b) \in \mathcal{R}$.

In the rest of the paper, we suppose that all the arguments from $\Sigma$ are constructed, i.e. that $\mathcal{A}=\operatorname{Arg}(\Sigma)$. We now introduce the notions of conflict-freeness and defence used to define different semantics.

Definition 3 (Conflict-free, defence). Let $\mathcal{F}=(\mathcal{A}, \mathcal{R})$ be an argumentation framework, $\mathcal{E} \subseteq \mathcal{A}$ and $a \in \mathcal{A}$.

$-\mathcal{E}$ is conflict-free if and only if there exist no two arguments $a, b \in \mathcal{E}$ s.t. $(a, b) \in \mathcal{R}$

$-\mathcal{E}$ defends a if and only if for every $b \in \mathcal{A}$ we have that if $b \mathcal{R} a$ then there exists $c \in \mathcal{E}$ such that $c \mathcal{R} b$.

Let us now define the most commonly used acceptability semantics.

Definition 4 (Acceptability semantics). Let $\mathcal{F}=(\mathcal{A}, \mathcal{R})$ be an argumentation framework and $\mathcal{B} \subseteq \mathcal{A}$. We say that a set $\mathcal{B}$ is admissible if and only if it is conflict-free and defends all its elements.

$-\mathcal{B}$ is a complete extension if and only if $\mathcal{B}$ defends all its arguments and contains all the arguments it defends.

$-\mathcal{B}$ is a preferred extension if and only if it is a maximal (with respect to set inclusion) admissible set.

$-\mathcal{B}$ is a stable extension if and only if $\mathcal{B}$ is conflict-free and for all $a \in \mathcal{A} \backslash \mathcal{B}$, there exists $b \in \mathcal{B}$ such that $b \mathcal{R}$ a. 
- $\mathcal{B}$ is a semi-stable extension if and only if $\mathcal{B}$ is a complete extension and the union of the set $\mathcal{B}$ and the set of all arguments attacked by $\mathcal{B}$ is maximal (for set inclusion).

$-\mathcal{B}$ is a grounded extension if and only if $\mathcal{B}$ is a minimal (for set inclusion) complete extension.

$-\mathcal{B}$ is an ideal extension if and only if $\mathcal{B}$ is a maximal (for set inclusion) admissible set contained in every preferred extension.

For an argumentation framework $\mathcal{F}=(\mathcal{A}, \mathcal{R})$ we denote by $\operatorname{Ext}_{x}(\mathcal{F})$; or, by a slight abuse of notation, by $\operatorname{Ext}_{x}(\mathcal{A}, \mathcal{R})$ the set of its extensions with respect to semantics $x$. We use abbreviations $c, p, s, s s, g$ and $i$ for respectively complete, preferred, stable, semi-stable, grounded and ideal semantics. For example, $\operatorname{Ext}_{p}(\mathcal{F})$ denotes the set of preferred extensions argumentation framework $\mathcal{F}$.

Example 2. Let $\mathcal{F}=(\mathcal{A}, \mathcal{R})$ be an argumentation framework with $\mathcal{A}=\{a, b, c, d\}$ and $\mathcal{R}=\{(b, c),(c, b),(b, d),(c, d)\}$. The graph is visualised below.

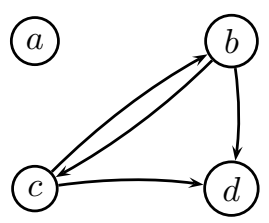

There are three complete extensions: $\{a, b\},\{a, c\}$ and $\{a\}$, and two preferred / stable / semi-stable extensions: $\{a, b\}$ and $\{a, c\}$. The grounded extension of this framework coincides with its ideal extension, which is the set $\{a\}$.

We now introduce the most common ways in which attack relations are defined in terms of (i.e. as functions of) the knowledge base in argumentation literature [9].

Definition 5. For a set of formulae $\Phi=\left\{\varphi_{1}, \ldots, \varphi_{k}\right\}$, let $\bigwedge \Phi$ denote $\varphi_{1} \wedge \ldots \wedge \varphi_{k}$. Let $a, b \in \operatorname{Arg}(\mathcal{L})$. We define the following attack relations:

- defeat: $a \mathcal{R}_{d} b$ if and only if $\operatorname{Conc}(a) \vdash \neg \bigwedge \operatorname{Supp}(b)$

- direct defeat: $a \mathcal{R}_{d d} b$ if and only if there exists $\varphi \in \operatorname{Supp}(b)$ s.t. $\operatorname{Conc}(a) \vdash \neg \varphi$

- undercut: $a \mathcal{R}_{u} b$ if and only if there exists $\Phi \subseteq \operatorname{Supp}(b)$ such that $\operatorname{Conc}(a) \equiv \neg \wedge \Phi$

- direct undercut: $a \mathcal{R}_{d u} b$ if and only if there exists $\varphi \in \operatorname{Supp}(b)$ s.t. $\operatorname{Conc}(a) \equiv \neg \varphi$

- canonical undercut: $a \mathcal{R}_{c u} b$ if and only if $\operatorname{Conc}(a) \equiv \neg \bigwedge \operatorname{Supp}(b)$

- rebut: $a \mathcal{R}_{r} b$ if and only if $\operatorname{Conc}(a) \equiv \neg \operatorname{Conc}(b)$

- defeating rebut: $a \mathcal{R}_{d r} b$ if and only if $\operatorname{Conc}(a) \vdash \neg \operatorname{Conc}(b)$

Note that all the attack relations from the previous definition are defined on $\operatorname{Arg}(\mathcal{L}) \times$ $\operatorname{Arg}(\mathcal{L})$. For a given $\Sigma$, one can just use the restriction of the relation from $\operatorname{Arg}(\mathcal{L}) \times$ $\operatorname{Arg}(\mathcal{L})$ to $\operatorname{Arg}(\Sigma) \times \operatorname{Arg}(\Sigma)$. This is not the case with all the attack relations we use in this paper. Namely, for some attack relations we use, there exist arguments $a, b \in \operatorname{Arg}(\mathcal{L})$, such that whether $a$ attacks $b$ or not depends also on the knowledge 
base $\Sigma$. Formally, the more general case is when an attack relation is defined by specifying its behaviour on every $\operatorname{Arg}(\Sigma) \times \operatorname{Arg}(\Sigma)$ for every finite $\Sigma \subseteq \mathcal{L}$. In the rest of the paper, when we use the term "attack relation", we refer to the more general case. Formally, one should write $(a, b, \Sigma) \in \mathcal{R}$. However, since it is always clear to which $\Sigma$ we refer to, there is no danger of confusion and in order to simplify the notation we write $(a, b) \in \mathcal{R}$ or $a \mathcal{R} b$ throughout the paper.

\section{Some Hypotheses Leading to Maximal Consistent Subsets of the Knowledge Base}

In this section, we identify four simple and intuitive conditions such that every instantiation which satisfies all of them returns the extensions corresponding exactly to maximal consistent subsets of the knowledge base arguments are constructed from. Namely, similar to the principles that can be satisfied by an acceptability semantics [4], there exist principles that an attack relation can satisfy $[1,5]$. An important requirement is that an attack relation should return consistent extensions (abbreviated CE).

Definition 6 (CE). Let $\mathcal{R}$ be an attack relation. We say that $\mathcal{R}$ returns consistent extensions under semantics $x$ if and only if for every $\Sigma \subseteq \mathcal{L}$, for every $\mathcal{F}=(\operatorname{Arg}(\Sigma), \mathcal{R})$, for every extension $\mathcal{E}$ of $\mathcal{F}$ under semantics $x$, it holds that $\operatorname{Base}(\mathcal{E})$ is a consistent set.

Example 3. $\mathcal{R}_{d u}$ and $\mathcal{R}_{d d}$ satisfy CE under stable, semi-stable, preferred, and complete semantics, whereas $\mathcal{R}_{u}, \mathcal{R}_{c u}, \mathcal{R}_{r}, \mathcal{R}_{d r}$ do not satisfy CE under neither of those semantics [9].

Another requirement in logic-based argumentation is that an argument should not attack another one if the union of their supports is consistent. This property of an attack relation is called conflict-dependence [1] for what we use the abbreviation CD.

Definition 7 (CD). Let $\mathcal{R}$ be an attack relation. We say that $\mathcal{R}$ is conflict-dependent if and only if for every $\Sigma \subseteq \mathcal{L}$, for every $a, b \in \operatorname{Arg}(\Sigma)$, if $(a, b) \in \mathcal{R}$ then $\operatorname{Supp}(a) \cup$ $\operatorname{Supp}(b) \vdash \perp$.

Example 4. Attack relations $\mathcal{R}_{d d}, \mathcal{R}_{u}, \mathcal{R}_{d u}, \mathcal{R}_{c u}, \mathcal{R}_{r}, \mathcal{R}_{d r}$ are conflict-dependent.

The next requirement specifies that the arguments having the same support are attacked by the same arguments. We call this AS ("assumption attack").

Definition 8 (AS). Let $\mathcal{R}$ be an attack relation. We say that $\mathcal{R}$ satisfies AS if and only if for every $\Sigma \subseteq \mathcal{L}$, for every $a, b, c \in \operatorname{Arg}(\Sigma)$, if $\operatorname{Supp}(b)=\operatorname{Supp}(c)$ then $a \mathcal{R} b$ if and

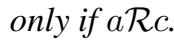

Note that AS is already present in argumentation literature [3, 9].

Example 5. Attack relations $\mathcal{R}_{d d}, \mathcal{R}_{u}, \mathcal{R}_{d u}$ and $\mathcal{R}_{c u}$ satisfy AS, whereas $\mathcal{R}_{r}$ and $\mathcal{R}_{d r}$ do not. 
The last property we consider in this paper specifies that when one constructs a set $\operatorname{Arg}(S)$ containing all the arguments made from a maximal consistent set $S$, then every argument outside of $\operatorname{Arg}(S)$ is attacked by at least one $\operatorname{argument}$ from $\operatorname{Arg}(S)$. We call the resulting condition MS, which is an abbreviation telling that the intuition behind it is that any maximal consistent set should be stable.

Definition 9 (MS). Let $\mathcal{R}$ be an attack relation. We say that $\mathcal{R}$ satisfies MS if and only if for every $\Sigma \subseteq \mathcal{L}$, for every $S \in \operatorname{MC}(\Sigma)$, for every $a^{\prime} \in \operatorname{Arg}(\Sigma) \backslash \operatorname{Arg}(S)$, there exists $a \in \operatorname{Arg}(S)$ such that $\left(a, a^{\prime}\right) \in \mathcal{R}$.

To the best of our knowledge, this property was not formally stated until now.

Example 6. Attack relations $\mathcal{R}_{d d}, \mathcal{R}_{u}, \mathcal{R}_{d u}, \mathcal{R}_{c u}$ satisfy MS.

Those conditions seem as properties one would like an attack relation to satisfy (at least in some contexts). The goal of this section is to answer the question: is it possible to define an instantiation of Dung's theory that captures reasoning substantially different from the approach which returns maximal consistent subsets and at the same satisfies CE, CD, AS and MS? The answer is no, as shown by Proposition 3.

We start by defining a notion of independence of a set of formulae, which is used to describe the extensions of attack relations satisfying AS. The idea is that no formula in a set can be derived from other formulae of that set.

Definition 10 (Independent set of formulae). A set $S \subseteq \mathcal{L}$ is independent if and only if there exists no formula $\varphi \in S$ s.t. $S \backslash\{\varphi\} \vdash \varphi$.

Our first goal is to show that for the class of attack relations satisfying AS, conclusion of an argument has no impact on its acceptability. In other words, the membership to an extension is uniquely determined by argument's support. To prove this result, we need the following lemma.

Lemma 1. Let $\mathcal{R}$ be an attack relation satisfying AS, let $\Sigma \subseteq \mathcal{L}$ be a knowledge base, $\mathcal{F}=(\operatorname{Arg}(\Sigma), \mathcal{R})$ and let $\mathcal{E} \subseteq \operatorname{Arg}(\mathcal{A})$ an admissible set. Let $a, b \in \operatorname{Arg}(\Sigma)$ be two arguments such that $\operatorname{Supp}(a)=\operatorname{Supp}(b), a \in \mathcal{E}$ and $b \notin \mathcal{E}$. Then, $\mathcal{E} \cup\{b\}$ is also an admissible set.

We can now show that if two arguments have the same support, and an attack relation satisfying AS is used, those two arguments are exactly in the same extensions.

Proposition 1. Let $\mathcal{R}$ be an attack relation satisfying AS, let $\Sigma \subseteq \mathcal{L}$ be a knowledge base, $\mathcal{F}=(\operatorname{Arg}(\Sigma), \mathcal{R})$ and $\mathcal{E} \in \operatorname{Ext}_{x}(\mathcal{F})$ with $x \in\{s, s s, p, g, i\}$. Let $a, b \in \operatorname{Arg}(\Sigma)$ and $\operatorname{Supp}(a)=\operatorname{Supp}(b)$. Then, $a \in \mathcal{E}$ if and only if $b \in \mathcal{E}$.

We can now show that for attack relations satisfying AS, every extension can be characterised by a collection of sets of formulae.

Proposition 2. Let $\mathcal{R}$ be an attack relation satisfying AS, let $\Sigma \subseteq \mathcal{L}$ be a knowledge base, $\mathcal{F}=(\operatorname{Arg}(\Sigma), \mathcal{R})$ and $\mathcal{E} \in \operatorname{Ext}_{x}(\mathcal{F})$ with $x \in\{s, s s, p, g, i\}$. Then: there exists a unique collection of sets $S_{1}, \ldots, S_{n} \subseteq \Sigma$ such that: 
1. every $S_{i}$ is consistent

2. every $S_{i}$ is independent

3. $\mathcal{E}=\left\{a \in \operatorname{Arg}(\mathcal{L}) \mid\right.$ there exists $S_{i}$ such that $\left.\operatorname{Supp}(a)=S_{i}\right\}$.

The significance of the previous result lays in the fact that it is a step forward towards understanding the expressivity of attack relations satisfying AS. Namely, is shows that every extension can be fully characterised by a unique collection of consistent and independent sets. Roughly speaking, every attack relation satisfying AS provides us with no more or less information than a function which separates $\Sigma$ in a finite number of collections of consistent and independent sets.

We can now prove that if an attack relation satisfies $\mathrm{CE}_{s}, \mathrm{CD}, \mathrm{AS}$ and MS, then its extensions are exactly the sets of arguments constructed from maximal consistent subsets of the knowledge base. In other words, for any maximal consistent subset $S$ of $\Sigma$, the set of all arguments constructed from $S$ is an extension, and for any extension, there exists a maximal consistent set $S \subseteq \Sigma$ such that $\mathcal{E}=\operatorname{Arg}(S)$.

Proposition 3. Let $\mathcal{R}$ be an attack relation satisfying $\mathrm{CE}_{s}, \mathrm{CD}, \mathrm{AS}$ and MS. Then, for every $\Sigma \subseteq \mathcal{L}$, extensions of $(\operatorname{Arg}(\Sigma), \mathcal{R})$ under stable semantics are exactly $\{\operatorname{Arg}(S)$ $\mid S \in \operatorname{MC}(\Sigma)\}$.

The previous result shows that the attack relations satisfying $\mathrm{CE}_{s}, \mathrm{CD}, \mathrm{AS}$ and $\mathrm{MS}$ simply mimic the result obtained by selecting the maximal consistent subsets of the knowledge base. This proposition is proved under stable semantics, but we believe that similar results can be obtained for other acceptability semantics, which will be a part of our future work.

\section{A New Class of Instantiations: Beyond Maximal Consistent Sets}

In this section, we show that if conditions AS and MS are dropped, it is possible to define a new instantiation of Dung's abstract argumentation theory which captures a result different from maximal consistent subsets of a knowledge base by and at the same time: i) uses only the information from the knowledge base (i.e. no external data about the preferences, values...), ii) and satisfies postulates from the argumentation literature (e.g. consistency, closure).

In general, it is possible to go from a knowledge base to a set of extensions in two steps. First, we define a measure, attaching to each element of a knowledge base a value; second, we define a procedure using that measure to calculate extensions. First, one can define different measures on the set of formulae of a propositional knowledge base. Second, once we have a measure, there are still many ways to go from the knowledge base and the measure to the sets of extensions. We can for example try to define an attack relation such that an extension contains the elements having a minimal sum of values. In this paper, we use the approach inspired by the work of Amgoud and Vesic [2]. The idea is to construct an attack relation which makes extensions contain as much elements having low values as possible, until a maximal consistent subset of a knowledge base is reached. 


\subsection{Shapley Inconsistency Value of a Formula}

The main idea behind the class of instantiations we propose is that the arguments made from "less inconsistent" formulae have "more chance" to be in extensions. This means that we need a tool for indicating how inconsistent a set or a formulae is. In this paper, we use Shapley Inconsistency Values, introduced by Hunter and Konieczny [10], to obtain that measure. This concept for measuring inconsistency is inspired by a Shapley Value, which was originally developed by Shapley [12] for defining merits of each individual of a coalition in a cooperative game theory.

The idea behind the class of instantiations we propose is that a user is free to choose a basic inconsistency measure, under the condition that it satisfies the four properties we state in the following definition. The corresponding Shapley Inconsistency Value can then be calculated automatically. Thus, different basic inconsistency measures give different Shapley Inconsistency Values.

Note that we present only the most important concepts linked to the definition of a Shapley Inconsistency Value, for more details the reader is referred to the paper in which they were introduced [10].

Definition 11 (Basic inconsistency measure [10]). A basic inconsistency measure I is a function that for every finite set of formulae returns a real number and satisfies the following properties for all finite sets $\Sigma, \Sigma^{\prime} \subseteq \mathcal{L}$ and all formulae $\varphi, \psi \in \mathcal{L}$ :

$-I(\Sigma)=0$ if and only if $\Sigma$ is a consistent set

(Consistency)

- $I\left(\Sigma \cup \Sigma^{\prime}\right) \geq I(\Sigma)$

(Monotony)

- If $\varphi$ is a free formula of $\Sigma \cup \varphi$, then $I(\Sigma \cup \varphi)=I(\Sigma)$ (Free Formula Independ.)

- If $\varphi \vdash \psi$ and $\varphi \nvdash \perp$, then $I(\Sigma \cup\{\varphi\}) \geq I(\Sigma \cup\{\psi\})$

(Dominance)

A basic inconsistency measure gives a number indicating how conflicting a knowledge base is. Let us give an example of a basic inconsistency measure.

Definition 12 (MI inconsistency measure [10]). The MI inconsistency measure is defined as the number of minimal inconsistent subsets of $\Sigma$, i.e.

$$
I_{\mathrm{MI}}(\Sigma)=|\operatorname{MinConf}(\Sigma)|
$$

Example 7. Let $\Sigma=\{\varphi, \neg \varphi, \varphi \rightarrow \psi, \neg \psi, \omega\}$. Then, $\operatorname{MinConf}(\Sigma)=\left\{C_{1}, C_{2}\right\}$, with $C_{1}=\{\varphi, \neg \varphi\}$ and $C_{2}=\{\varphi, \varphi \rightarrow \psi, \neg \psi\}$. Thus, $\operatorname{MI}(\Sigma)=2$.

The MI inconsistency measure is a basic inconsistency measure.

Originally, Shapley's idea was to measure the merit of an individual in a coalition. Here, the idea is to use it to measure the "blame" of a formula for the inconsistency of a knowledge base. To do that, the identical mathematical expression from Shapley [12] is used, but with different interpretation.

Definition 13 (Shapley Inconsistency Value [10]). Let $\Sigma \subseteq \mathcal{L}$ and let I be a basic inconsistency measure. We define the corresponding Shapley Inconsistency Value (SIV), noted $S^{I}$, as follows. For every $\varphi \in \Sigma$ :

$$
S_{\varphi}^{I}(\Sigma)=\sum_{S \subseteq \Sigma} \frac{(|S|-1) !(|\Sigma|-|S|) !}{|\Sigma| !}(I(S)-I(S \backslash\{\varphi\})) .
$$


Beside the fact that this measure gives very sensible results, it has also been shown that the previous formula is the only one which satisfies a set of intuitive axioms for measuring inconsistency [10]. This SIV gives a value for each formula of the base $\Sigma$. Thus, the previous definition allows us to define to what extent a formula is concerned with the inconsistencies. Note that for a formula $\varphi$, SIV depends essentially on the sum of differences of inconsistencies of all subsets of $\Sigma$ together and without $\varphi$. Those values are then just multiplied with coefficients which depend only on the cardinalities of the corresponding sets. So, the main intuition can be resumed in: "How much does inconsistency decrease when $\varphi$ is removed?"

It has been shown [10] that the SIV corresponding to basic inconsistency measure MI is:

$$
S_{\varphi}^{I_{M I}(\Sigma)=} \sum_{C \in \operatorname{MinConf}(\Sigma) \text { such that } \varphi \in C} \frac{1}{|C|} .
$$

In other words, the inconsistency blame of a formula $\varphi$ is obtained by summing up the values $\frac{1}{|C|}$ for all minimal conflicts $C$ such that $\varphi \in C$.

Example 8 (Example 7 Cont.). SIV values of the formulae from $\Sigma$ are as follows: $S_{\varphi}^{I_{M I}}(\Sigma)=\frac{5}{6}, S_{\neg \varphi}^{I_{M I}}(\Sigma)=\frac{1}{2}, S_{\varphi \rightarrow \psi}^{I_{M I}}(\Sigma)=\frac{1}{3}, S_{\neg \psi}^{I_{M I}}(\Sigma)=\frac{1}{3}$, and $S_{\omega}^{I_{M I}}(\Sigma)=0$.

On the one hand, this measure takes into account the fact that a formula being in more minimal inconsistent sets is more inconsistent (which can be justified by saying that to obtain consistency, one has to remove at least one formula from every minimal conflict, thus by removing a formula which is in more minimal conflicts, one obtains consistency "faster"). On the other hand, this measure takes into account the intuition that, for example, a formula is in a minimal inconsistent set having 1000 formulae makes it "less inconsistent" than if it were in a minimal inconsistent sets having 2 formulae.

However, MI is just one possible basic inconsistency value, which we presented in order to illustrate the idea. In the rest of the paper, we suppose that an arbitrary basic inconsistency measure and the corresponding SIV are used.

\subsection{Defining Instantiations}

In this section, we use the method for measuring inconsistency of a formula to define an instantiation of Dung's abstract argumentation theory. Suppose that we are given a basic inconsistency measure. We can obtain the corresponding SIV, and use it to compare the formulae of the knowledge base. We first define how to construct a stratified version of a knowledge base, where the least inconsistent formulae (according to a given measure) are put in $\Sigma_{0}$ and the most inconsistent ones in $\Sigma_{n}$.

Definition 14 (Inconsistency ordered version of a knowledge base). Let I be a basic inconsistency measure, and $S^{I}$ the corresponding SIV. Let $\Sigma \subseteq \mathcal{L}$ be a knowledge base. The inconsistency ordered version of $\Sigma$ (with respect to $I$ ) is a n-tuple $\left(\Sigma_{0}, \ldots, \Sigma_{n}\right)$ such that 
- $\Sigma_{0} \cup \ldots \cup \Sigma_{n}=\Sigma$,

- for every $i, j \in\{0, \ldots, n\}$, if $i \neq j$ then $\Sigma_{i} \cap \Sigma_{j}=\emptyset$,

- for any two formulae $\varphi, \psi \in \Sigma$ such that $\varphi \in \Sigma_{i}$ and $\psi \in \Sigma_{j}$, we have

$$
S_{\varphi}^{I}(\Sigma) \geq S_{\psi}^{I}(\Sigma) \text { if and only if } i \geq j .
$$

Example 9 (Example 8 Cont.). The inconsistency ordered version of $\Sigma$ with respect to MI is: $\Sigma_{0}=\{\omega\}, \Sigma_{1}=\{\varphi \rightarrow \psi, \neg \psi\}, \Sigma_{2}=\{\neg \varphi\}, \Sigma_{3}=\{\varphi\}$.

This order induces a preference on $\Sigma$, which can be used to define a preference relation on $\operatorname{Arg}(\Sigma)$. Let us first define a level of a formula and of an argument.

Definition 15 (Level of formulae and arguments). Let I be a basic inconsistency measure, $S^{I}$ the corresponding SIV, let $\Sigma \subseteq \mathcal{L}$ be a knowledge base and $\left(\Sigma_{0}, \ldots, \Sigma_{n}\right)$ its inconsistency ordered version with respect to I. For a formula $\varphi \in \Sigma$,

$$
\operatorname{level}(\varphi)=i \text { if and only if } \varphi \in \Sigma_{i} .
$$

For an argument $a \in \operatorname{Arg}(\Sigma)$,

$$
\operatorname{level}(a)=\max _{\varphi \in \operatorname{Supp}(a)} \operatorname{level}(\varphi) .
$$

We can now define an attack relation taking into account the level of formulae.

Definition 16 (Direct undercut on the ordered knowledge base). Direct undercut on the ordered knowledge base $\left(\Sigma_{0}, \ldots, \Sigma_{n}\right)$ is a relation $\mathcal{R}_{\text {duo }}$ defined as: a $\mathcal{R}_{\text {duo }} b$ if and only if $\left(a \mathcal{R}_{d u} b\right.$ and level $\left.(a) \leq \operatorname{level}(b)\right)$ or $\left(b \mathcal{R}_{d u} a\right.$ and level $\left.(a)<\operatorname{level}(b)\right)$.

As an illustration we consider again our running example.

Example 10 (Example 9 Cont.). Let $a=(\{\neg \psi, \varphi \rightarrow \psi\}, \neg \varphi), b=(\{\varphi\}, \varphi)$, and $c=$ $(\{\neg \varphi\}, \neg \varphi)$. Then, $a \mathcal{R}_{d u} b$, level $(a)=1$ and level $(b)=3$. Thus, $a \mathcal{R}_{d u o} b$. However, even if $b \mathcal{R}_{d u} c$, we do not have that $b \mathcal{R}_{d u o} c$, since level $(b)=3$ and level $(c)=2$.

Attack relation $\mathcal{R}_{d u o}$ satisfies CD.

Proposition 4. For any basic inconsistency measure I and the corresponding SIV $S^{I}$, $\mathcal{R}_{\text {duo }}$ is $\mathrm{CD}$.

We can also show that it returns consistent extensions which are closed for $\vdash$ and for sub-arguments ${ }^{1}$.

Proposition 5. Let I be a basic inconsistency measure and $S^{I}$ the corresponding Shapley inconsistency measure. Let $\Sigma \subseteq \mathcal{L}$ be a knowledge base and $\left(\Sigma_{0}, \ldots, \Sigma_{n}\right)$ its inconsistency ordered version. Let $\mathcal{E}$ be a stable extension of $\left(\operatorname{Arg}(\Sigma), \mathcal{R}_{d u o}\right)$. Then:

- Base $(\mathcal{E})$ and $\operatorname{Concs}(\mathcal{E})$ are consistent sets

- $\operatorname{Con} \operatorname{cs}(\mathcal{E})$ is closed for $\vdash$, i.e. for every $\varphi \in \mathcal{L}$, if $\operatorname{Concs}(\mathcal{E}) \vdash \varphi$ then $\varphi \in$ $\operatorname{Concs}(\mathcal{E})$,

\footnotetext{
${ }^{1}$ We suppose the definition of sub-argument by Gorogiannis and Hunter [9].
} 
$-\mathcal{E}$ is closed for sub-arguments, i.e. if $a \in \mathcal{E}$ and $b$ is an argument such that $\operatorname{Supp}(b) \subseteq$ $\operatorname{Supp}(a)$, then $b \in \mathcal{E}$.

Note that by following the approach we describe in this section, one obtains a refinement of the approach returning extensions corresponding to the maximal consistent subsets of the knowledge base. Namely, if a basic inconsistency measure is used to order the knowledge base, and $\mathcal{R}_{d u o}$ is then applied to calculate the extensions under stable semantics, every extension corresponds to exactly one maximal consistent subset of $\Sigma$, but there are some maximal consistent subsets of $\Sigma$ which do not correspond to any extensions. Proposition 6 shows that for every extension, there exists a maximal consistent subset of $\Sigma$ corresponding to that extension. Example 11 illustrates the fact that there can exist maximal consistent sets which do note correspond to any stable extensions.

Proposition 6. Let I be a basic inconsistency measure and $S^{I}$ the corresponding SIV. Let $\Sigma \subseteq \mathcal{L}$ be a knowledge base and $\left(\Sigma_{0}, \ldots, \Sigma_{n}\right)$ its inconsistency ordered version. Then:

$$
\operatorname{Ext}_{s}\left(\left(\operatorname{Arg}(\Sigma), \mathcal{R}_{\text {duo }}\right)\right) \subseteq\{\operatorname{Arg}(S) \mid S \in \operatorname{MC}(\Sigma)\}
$$

Example 11 (Example 10 Cont.). The set $S=\{\varphi, \varphi \rightarrow \psi, \omega\}$ is a maximal consistent subset of $\Sigma$. Let $d=(\{\neg \psi\}, \neg \psi)$. It is clear that $d \notin \operatorname{Arg}(S)$. However, no argument from $\operatorname{Arg}(S)$ attacks $d$ with respect to $\mathcal{R}_{d u o}$. There exists only one argument $e=(\{\varphi, \varphi \rightarrow \psi\}, \psi)$, such that $e \in \operatorname{Arg}(S)$ and $e \mathcal{R}_{d u} d$, but level $(e)>\operatorname{level}(d)$, thus $e$ is more inconsistent than $d$ and, according to the definition of $\mathcal{R}_{d u o}$, does not attack $d$.

We see from Propositions 4 and 5 that $\mathcal{R}_{d u o}$ satisfies $\mathrm{CD}$ and $\mathrm{CE}_{s}$. We now show that this attack relation falsifies AS and MS. To show that $\mathcal{R}_{d u o}$ falsifies AS, consider the following example.

Example 12. Let $\Sigma=\{\neg \varphi, \neg(\varphi \wedge \psi), \varphi \wedge \psi\}$, and let us use the MI inconsistency measure and the corresponding Shapley Inconsistency Value $S^{I_{M I}}$. Then, $\Sigma_{0}=\{\neg \varphi, \neg(\varphi \wedge$ $\psi)\}$ and $\Sigma_{1}=\{\varphi \wedge \psi\}$. Let $a=(\{\neg \varphi\}, \neg \varphi), b=(\{\varphi \wedge \psi\}, \varphi)$, and $c=(\{\varphi \wedge \psi\}, \psi)$. Then, $\operatorname{Supp}(b)=\operatorname{Supp}(c)$, but at the same time $a \mathcal{R}_{d u o} b$ and $\neg\left(a \mathcal{R}_{d u o} c\right)$. Thus, $\mathcal{R}_{\text {duo }}$ does not satisfy AS.

By examining Example 10 one can observe that no argument attacks argument $c=$ $(\{\neg \varphi\}, \neg \varphi)$ in this example. Thus, $\mathcal{R}_{d u o}$ does not satisfy MS.

\section{Summary}

This paper advances the state of the art in instantiating Dung's abstract argumentation theory in several ways. First, we identify four simple conditions describing a wide class of attack relations based on attacking premises of an argument which return extensions corresponding to exactly maximal consistent subsets of the propositional knowledge base. Second, we show that when two of the conditions are dropped, it is possible to instantiate Dung's abstract argumentation theory with classical propositional logic and 
to obtain a result substantially different from the extensions which correspond to maximal consistent subsets of the knowledge base, without having external information such as preferences or values. We use Shapley Inconsistency Values [10] to measure inconsistency of a particular formula in the knowledge base and use that value to define attack relations which select extensions made of less inconsistent formulae. Third, we show that this whole class of instantiations satisfies the usual rationality postulates: its extensions have consistent bases, they are closed for sub-arguments, etc.

We identified a new class of inference relations that can be captured in Dung's theory, which is a first step towards a better understanding of possibilities and constraints imposed by this abstract theory. Our next goal is to characterise the class of all inference relations that can be represented in such a way.

To capture different results from simply returning the extensions corresponding to maximal consistent sets, we use an original attack relation, which has several features deserving some comments. First, this attack relation is dependent on the knowledge base $\Sigma$. In other words, whether an argument attacks another one cannot be determined without knowing what knowledge base they come from. This raises some conceptually and technically interesting questions which will be part of our future work. Second, the procedure we use rank-orders arguments on the basis of some kind of preference on the formulae in their supports. Our attack relation in some way "simulates" what is done in preference-based argumentation [2], and protects less inconsistent arguments from more inconsistent ones. An important difference is that in the present paper, we do not suppose any preferences at the input of our system. If the proposed class of instantiations selects some maximal consistent sets and not all of them, it comes from the fact that they have different degrees of inconsistency.

Obviously, the result of our work depends on the acceptability semantics used for evaluating arguments. Our main results were shown under stable semantics. We plan to examine whether similar results can be obtained under other semantics, and more generally, to determine the role played by a semantics when capturing different results as instantiations of Dung's abstract theory. Our goal is to study a large class of semantics satisfying some minimal requirements [4] (e.g. conflict-freeness, syntax independence).

This paper shows that the class of attack relations satisfying CE, CD, AS and MS is rather narrow, in the sense that they always return a result identical to that obtained from maximal consistent sets of the knowledge base. Thus, if one wants to subsume richer approaches, at least one of those four conditions has to be dropped. For example, Section 4 of the current paper uses attack relations satisfying CD and CE and violating AS and MS. First, note that we present the first attack relation which violates AS and returns sound results. Considering violating MS, it does not seem surprising, since this condition basically says that every maximal consistent set should yield a stable extension. Violating conflict-dependency and keeping some good properties of the system looks like a difficult task, although we do not claim that is impossible. However, it would be hard to justify attack relations returning extensions with inconsistent bases. The only possible explanation for that could be that argumentation is seen just as the first step of some longer process, and it resolves some (but not necessarily all) conflicts. Then, another mechanism is used to reason with the set of obtained extensions. 


\section{References}

1. L. Amgoud and P. Besnard. Bridging the gap between abstract argumentation systems and logic. In International Conference on Scalable Uncertainty Management (SUM'09), pages 12-27, 2009.

2. L. Amgoud and S. Vesic. A new approach for preference-based argumentation frameworks. Annals of Mathematics and Artificial Intelligence, 2011.

3. L. Amgoud and S. Vesic. On the equivalence of logic-based argumentation systems. In Proceedings of the 5th International Conference on Scalable Uncertainty Management, (SUM'11), pages 123-136. Springer, 2011.

4. P. Baroni and M. Giacomin. On principle-based evaluation of extension-based argumentation semantics. Artificial Intelligence Journal, 171:675-700, 2007.

5. M. Caminada and L. Amgoud. On the evaluation of argumentation formalisms. Artificial Intelligence Journal, 171 (5-6):286-310, 2007.

6. C. Cayrol. On the relation between argumentation and non-monotonic coherence-based entailment. In Proceedings of the 14th International Joint Conference on Artificial Intelligence (IJCAI'95), pages 1443-1448, 1995.

7. P. M. Dung. On the acceptability of arguments and its fundamental role in nonmonotonic reasoning, logic programming and $n$-person games. Artificial Intelligence Journal, 77:321357, 1995.

8. D. Gabbay, G. Pigozzi, and O. Rodrigues. Common foundations for belief revision, belief merging and voting. In Formal Models of Belief Change in Rational Agents (Dagstuhl Seminar Proceedings), 2007.

9. N. Gorogiannis and A. Hunter. Instantiating abstract argumentation with classical logic arguments: Postulates and properties. Artificial Intelligence Journal, 175:1479-1497, 2011.

10. A. Hunter and S. Konieczny. On the measure of conflicts: Shapley inconsistency values. Artificial Intelligence Journal, 174(14):1007-1026, 2010.

11. G. Priest. Paraconsistent logic. In D. Gabbay and F. Guenthner, editors, Handbook of Philosophical Logic, volume 6, pages 287-393. Dordrecht: Kluwer Academic Publishers, 2002.

12. L. Shapley. A value for n-person games. In Contributions to the Theory of Games II, pages 307-317. Princeton University Press, 1953. 